\title{
PRACTICAL CREDIBILITY THEORY WITH EMPHASIS ON OPTIMAL PARAMETER ESTIMATION
}

\section{F. DE VYLDER*}

We develop Hachemeister's regression model in credibility theory (without proofs) and indicate how the involved structural parameters can be estimated from the observable variables (with proofs for the simple results and those not yet published).

Large families of unbiased estimators are available. From the practical viewpoint this is rather a handicap because it creates the problem to decide what estimators actually to use. In order to fix optimal estimators, we adopt the small-sample criterion of minimum-variance. But in the research for general solutions three kinds of difficulties arise.

(i) The calculations become too lengthy.

(ii) The optimal estimators depend on some of the parameters to be estimated. (Then we call them pseudo-estimators).

(iii) The optimal estimators depend on new structural parameters defined in terms of fourth-order moments.

Only a compromise allows to cope with this reality. Situation (iii) creates new estimation problems. They can only be avoided at the cost of the introduction of special assumptions or approximations. Then problem (i) is more or less automatically solved. By an obvious method of successive approximations pseudo-estimators can serve as true estimators. Thus (ii) is no real problem.

\section{General notations and Definitions}

\subsection{Matrices}

The same main symbol is used to denote a matrix $c$ and its elements $c_{i}^{j}$. Here $c_{i}^{j}$ is the element at the intersection of row $i$ and column $j$. The row $i$ is denoted by $c_{i}$ and the column $j$ by $c^{j}$. When the number of rows is $m$ and the number of columns $n$, we say that the matrix has dimensions $\left(\begin{array}{l}n \\ m\end{array}\right)$.

These conventions are most convenient for automatical calculation with matrices. For instance, if

then

$$
\begin{aligned}
& a=b \quad c \quad d \\
& \left(\begin{array}{l}
\mathbf{n} \\
\mathbf{m}
\end{array}\right) \quad\left(\begin{array}{l}
\mathbf{p} \\
\mathbf{m}
\end{array}\right)\left(\begin{array}{l}
\mathbf{q} \\
\mathbf{p}
\end{array}\right)\left(\begin{array}{l}
\mathbf{n} \\
\mathbf{q}
\end{array}\right)
\end{aligned}
$$

$$
a_{i}=b_{i} c d, a^{j}=b c d^{j}, a_{i}^{j}=b_{i} c d^{j}, a=\underset{r}{\sum} b^{r} c_{r} d, a_{i}=\sum_{r, s} b_{i}^{r} c_{r}^{s} d_{s}, \ldots
$$

* The author is thankful to the referee who improved this paper. 
Hence the following general rules in case of a matrix relation such as (1), with any number of factors in the last member:

(i) The same lower index can be placed at the matrix in the first member and at the first matrix in the last member.

(ii) The same upper index can be placed at the matrix in the first member and the the last matrix in the last member.

(iii) In the last member, an upper index can be placed at any matrix and the same lower index at the following matrix, provided that index be summed out.

Of course, according to the same rules applied in the opposite direction, indices can also be dropped.

We recall that the trace of a square matrix $c$ is the sum of its diagonal elements: $\operatorname{tr} c=\Sigma c_{i}^{i}$. When a product, say $a b c d$, of any number of any matrices is a square matrix, then any cyclical permutation, say $c d a b$, is also a square matrix (of possibly different dimensions) and $\operatorname{tr}(a b c d)=\operatorname{tr}(c d a b)$.

\subsection{Random matrices}

The expectation $E A$ of a matrix $A$ with random elements $A_{i}^{j}$ is the matrix with elements $E A_{i}^{j}$. The operators tr and $E$ commute.

To the $\left(\begin{array}{l}1 \\ n\end{array}\right)$ random column $X$ is associated the $\left(\begin{array}{l}1 \\ n\end{array}\right)$ covariance matrix Cov $X$ with elements

$$
(\operatorname{Cov} X)_{i}^{j}=\operatorname{Cov}\left(X_{i}, X_{j}\right)=E\left(X_{i} X_{j}\right)-E\left(X_{i}\right) E\left(X_{j}\right)
$$

Using the accent for transposition, the last member can be displayed as $E\left(X_{i} X^{\prime f}\right)-E\left(X_{i}\right) E\left(X^{\prime j}\right)$. Therefore

$$
\operatorname{Cov} X=E\left(X X^{\prime}\right)-E(X) E\left(X^{\prime}\right),\left(\begin{array}{l}
n \\
n
\end{array}\right) \text {. }
$$

When $X$ is $\left(\begin{array}{l}1 \\ 1\end{array}\right)$, then $\operatorname{Cov} X$ is in fact $\operatorname{Var} X$.

If $t$ is $\left(\begin{array}{l}n \\ m\end{array}\right)$ and $X\left(\begin{array}{l}1 \\ n\end{array}\right)$, then $t X$ is $\left(\begin{array}{c}1 \\ m\end{array}\right)$ and $\operatorname{Cov}(t X)=t(\operatorname{Cov} X) t^{\prime}$

Similarly, for the conditional covariance matrix:

$$
\operatorname{Cov}(X / \Theta)=E\left(X X^{\prime} / \Theta\right)-E(X / \Theta) E\left(X^{\prime} / \Theta\right),\left(\begin{array}{l}
n \\
n
\end{array}\right)
$$

The following relation is easy:

$$
\operatorname{Cov} X=E \operatorname{Cov}(X / \Theta)+\operatorname{Cov} E(X / \Theta),\left(\begin{array}{l}
n \\
n
\end{array}\right) .
$$

We define the scalar variance of any random matrix $A$ by

$$
\begin{aligned}
\operatorname{ScaVar} A & =\sum_{i, j} \operatorname{Var} A_{j}^{i}=\sum_{i, j} E\left(A_{i}^{j} A_{j}^{\prime i}\right)-\sum_{i, j} E(A) E\left(A_{j}^{\prime i}\right) \\
& =\sum_{i} E\left(A_{i} A^{\prime} i\right)-\sum_{i} E\left(A_{i}\right) E\left(A^{\prime} i\right)=\operatorname{tr} E\left(A A^{\prime}\right)-\operatorname{tr}\left(E A . E A^{\prime}\right) .
\end{aligned}
$$


Thus, when $A$ is the $\left(\begin{array}{l}1 \\ n\end{array}\right)$ column $X$, then ScaVar $X=\operatorname{tr} \operatorname{Cov} X$.

To say that the $\left(\begin{array}{l}1 \\ n\end{array}\right)$ random vector $X$ is $N(m, v)$ means that $X$ is normally distributed with $E X=m, \operatorname{Cov} X=v$. This implies that $m$ is $\left(\begin{array}{l}1 \\ n\end{array}\right)$ and that $v$ is $\left(\begin{array}{l}n \\ n\end{array}\right)$. Similarly, "the conditional vector $X$, given $\Theta$, is $N(m(\Theta)$, $v(\Theta))$ " means that this vector is normally distributed with $E(X / \Theta)=m(\Theta), \operatorname{Cov}(X / \Theta)=v(\Theta)$.

\subsection{Miscellaneous}

In case of multiple indices, the matrix rules are applied to the last indices only. For instance, if the scalars $x_{j}^{q} p$ are defined for variable $j, p, q$, then, for each fixed $j, x_{j}$ is the matrix with elements $x_{j p}^{q}, x_{j p}$ is the $p$-th row of that matrix and $x_{j}^{q}$ is its $q$-th column. These notations are unambiguous if the reader keeps in mind the number and positions of the subscripts on the initially considered scalars. Thus, in case of the just considered $x_{j p}, x_{j}, x_{j p}, x_{j}^{q}$ it must be remembered that the scalars $x_{j p}^{q}$ have two subscripts and one superscript.

For any finite sequence $x_{i}$ of square matrices, we define

$$
x_{\Sigma}=\Sigma x_{i}, \bar{x}_{i}=x_{\Sigma}^{-1} x_{i},
$$

provided $x_{\Sigma}^{-1}$ exists, for the last relation. The sequence $\bar{x}_{i}$ is the pre-normed sequence $x_{i}$. In summations, all indices, matricial or not, must be completely summed out, unless stated otherwise.

If $A$ is a random matrix, we denote by $A^{\circ}$ the centered matrix $A^{\circ}=A-$ $E A$. No confusions can arise between ${ }^{\circ}$ and a matrix index, because ${ }^{\circ}$ shall never be used as a matrix index.

2. HACHEMEISTER'S REgRESSION MODEL. (HACHEMEISTER, 1975)

\subsection{Definition of the model}

We consider the array of observable random variables

$$
\begin{array}{llll}
X_{11} & X_{21} & \ldots & X_{k 1} \\
X_{12} & X_{22} & \ldots & X_{k 2} \\
\ldots & \ldots & \ldots & \ldots \\
X_{1 t} & X_{2 t} & \ldots & X_{k t}
\end{array}
$$

The class $j$ or risk $j$ is the $\left(\begin{array}{l}1 \\ t\end{array}\right)$ column $X^{j}$ in that array.

The index $s$ in $X_{j s}$ is interpreted as a time index. To $X_{j}$ is associated the structure variable $\Theta_{j}$ (possibly multi-dimensional).

The possible values for $i, j$ are always $i, j=1,2, \ldots, k$. It is assumed that all quantities further displayed actually exist and are finite.

The following assumptions are made.

(i) Independence of completed classes: the couples $\left(X_{1}, \Theta_{1}\right),\left(X_{2}, \Theta_{2}\right), \ldots$, $\left(X_{k}, \Theta_{k}\right)$ are independent. 
(ii) Equidistribution of structure variables: the random variables $\Theta_{1}, \Theta_{2}, \ldots$, $\Theta_{k}$ are identically distributed.

(iii) Regression assumption: $E\left(X_{j} / \Theta_{j}\right)=y \beta\left(\Theta_{j}\right),\left(\begin{array}{l}\mathbf{1} \\ t\end{array}\right)$, where

$y$ is a given $\left(\begin{array}{l}g \\ t\end{array}\right)$ matrix of full rank $g<t$,

$\beta($.$) is a \left(\begin{array}{l}1 \\ g\end{array}\right)$ vector of unknown functions $\beta_{p}($.$) .$

(iv) Covariance assumption: $\operatorname{Cov}\left(X_{j} / \Theta_{j}\right)=\sigma^{2}\left(\Theta_{j}\right) v_{j}$, $\left(\begin{array}{l}t \\ t\end{array}\right)$, where

$\sigma^{2}($.$) is an unknown scalar function,$

$v_{j}$ is a given positive definite $\left(\begin{array}{l}t \\ t\end{array}\right)$ matrix.

\subsection{Interpretations and problem}

The meaning of the observable random variables $X_{j s}$ and of the matrices $y, v_{j}$ is exemplified in Hachemeister (1975).

At a first stage, it is imagined that the distribution of $X_{j}$ depends on a parameter $\theta_{j}$. Because it is unknown, this parameter is interpreted as a realization of some hidden random variable $\Theta_{j}$.

The actuary is interested in $E\left(X_{j} / \Theta_{j}\right)$ or equivalently, because of the regression assumption, in $\beta_{p}\left(\Theta_{j}\right),(p=1,2, \ldots, g)$. Usually however, $\beta_{p}($.$) is$ unknown and $\Theta_{j}$ cannot be observed. Therefore, the actuary replaces his problem by a simpler one. He approximates $\beta_{p}\left(\Theta_{j}\right)$ by a linear expression.

$$
B_{j p}=a_{\mathrm{o}}+\sum_{i s} a_{i s} X_{i s}
$$

of the observable variables $X_{j s}$. The unknown coefficients $a_{\mathbf{0}}$, $a_{i s}$ (depending on $j, p)$ are fixed in such a way that

$$
E\left(\beta_{p}\left(\Theta_{j}\right)-B_{j p}\right)^{2}
$$

becomes minimum. The so obtained $\left(\begin{array}{l}1 \\ g\end{array}\right)$ vector $B_{j}$ is the credibility estimator for $\beta\left(\Theta_{j}\right)$.

\subsection{Solution of the problem (SEe Hachemeister 1975 OR DE VyLder 1976)}

The credibility estimator $B_{j}$ for $\beta\left(\Theta_{j}\right)$ equals

$$
B_{j}=\left(1-z_{j}\right) b+z_{j} \hat{b}_{j},\left(\begin{array}{l}
1 \\
g
\end{array}\right),
$$

where

$$
\begin{gathered}
z_{j}=a\left(a+s^{2} w_{j}\right)^{-1}\left(\begin{array}{l}
g \\
g
\end{array}\right), w_{j}=\left(y^{\prime} v_{j}^{-1} y\right)^{-1}\left(\begin{array}{l}
g \\
g
\end{array}\right), \\
\hat{b}_{j}=\left(y^{\prime} v_{j}^{-1} y\right)^{-1} y^{\prime} v_{j}^{-1} X_{j},\left(\begin{array}{l}
1 \\
g
\end{array}\right)
\end{gathered}
$$

and where

$$
a=\operatorname{Cov} \beta\left(\Theta_{j}\right)\left(\begin{array}{l}
g \\
g
\end{array}\right), b=E \beta\left(\Theta_{j}\right)\left(\begin{array}{l}
1 \\
g
\end{array}\right), s^{2}=E \sigma^{2}\left(\Theta_{j}\right) .
$$

With Norberg (1979), we call $a, b, s^{2}$ structural parameters of the model. Briefly we shall call $a, b, s^{2}$ the covariance matrix, the mean vector, the variance 
respectively. The matrix $z_{j}$ is the credibility matrix for class $j$. When $g=1$, all these quantities are scalars.

\section{The general problem of structural parameter estimation}

\subsection{Small-sample properties of the estimators}

Unbiasedness and minimum-variance shall be considered as most wanted properties of the estimators for the structural parameters. These are smallsample properties.

Asymptotical properties of some estimators can also be proved. For instance see Norberg $(1979,1980)$.

Such properties become only interesting in case of large samples of observed random variables. How large? This certainly depends on the complexity of the involved model. In case of Hachemeister's model, no precise answer to the question can be expected soon.

\subsection{Families of unbiased estimators}

In DE VYLDER (1978), we proposed families of unbiased estimators for the structural parameters. Here we shall propose the same families for $b$ and $s^{2}$ but a different one for $a$.

\subsection{Minimum-variance estimators}

The minimum-variance estimator in a family of estimators $\hat{a}\left(\hat{b}, \hat{s}^{2}\right)$ for $a\left(b, s^{2}\right)$ is that one making ScaVar $\hat{a}$ (ScaVar $\hat{b}$, Var $\hat{s}^{2}$ ) minimum. When looking for such minimum-variance estimators, one may have the sad surprise that the estimators contain some of the structural parameters to be estimated. However, that situation is far from hopeless as we shall see in 3.4 .

\subsection{Pseudo-statistics and pseudo-estimators}

We call pseudo-statistic any known function of the observable random variables and of the structural parameters. Similarly, psendo-estimators may depend on the parameters to be estimated. Such pseudo-estimators may nevertheless work as true estimators. For instance, consider the pseudo-estimators

$$
\hat{a}=f\left(X_{11}, \ldots, X_{k t}, a, b\right), \hat{b}=g\left(X_{11}, \ldots, X_{k t}, a, b\right)
$$

for $a, b$. Suppose that the last members in (6) are not too sensitive to small variations of $a, b$. Then rather arbitrary initial estimates $a(o), b(0)$ for $a, b$ can be used in (6), furnishing first approximations $a(1), b(1)$. The latter can be used again in (6), furnishing second approximations $a(2), b(2)$ and so on. When practical convergence of the sequences $a(n), b(n)$ is observed, the practical limits can be considered as final estimates for $a, b$.

Of course it amounts to the same to solve the system of equations 


$$
a=f\left(X_{11}, \ldots, X_{k t}, a, b\right), b=g\left(X_{11}, \ldots, X_{k t}, a, b\right)
$$

in the unknown quantities $a, b$.

Pseudo-estimators are used since several years by Dr. F. Bichsel in unpublished practical work.

\section{Estimation of the Mean vector $b$}

\subsection{Fixed-class estimator}

From the regression assumption results: $E X_{j}=y b$. Then $E \hat{b}_{j}=b$ by (4). This means that $\hat{b}_{j}$ is an unbiased estimator for $b$. In fact $b_{j}$ is the classical least squares estimator, in class $j$, for $b$.

\subsection{Family of unbiased pseudo-estimators}

It follows that

$$
\hat{b}=\Sigma x_{j} \hat{b}_{j}\left(\begin{array}{l}
1 \\
g
\end{array}\right),\left(x_{j}\left(\begin{array}{l}
g \\
g
\end{array}\right), x_{\Sigma}=1\right)
$$

is an unbiased estimator of $b$. Since we do not exclude that the $x_{j}$ contain some structural parameters, relation (8) defines in fact a family of pseudoestimators for $b$.

\subsection{Minimum-variance pseudo-estimator}

In DE VYLDER (1978), it is proved that the minimum-variance pseudoestimator in the family (8) is obtained when $x_{j}$ is the credibility matrix $z_{j}$ pre-normed, i.e. $x_{j}=\bar{z}_{j}$. This minimum-variance estimator is denoted by (9)

$$
\widehat{\hat{b}}=\Sigma \bar{z}_{j} \hat{b}_{j}
$$

It is noteworthy that TAYLOR (1977) and, in a particular case, BÜHLMANN and StRAUB (1970) obtained this optimal estimator automatically after the introduction of suitable constraints.

\section{Estimation of the variance $s^{2}$}

\subsection{Fixed-class estimator}

The classical estimator for $s^{2}$, in class $j$, is

$$
\hat{s}_{j}^{2}=\frac{1}{t-g}\left(X_{j}-y \hat{b}_{j}\right)^{\prime} v_{j}^{-1}\left(X_{j}-y \hat{b}_{j}\right) .
$$

It is unbiased. See De VyLDeR (1978).

\subsection{Family of unbiased estimators}

It follows that

$$
\hat{s}^{2}=\Sigma x_{j} \hat{s}_{j}^{2},\left(x_{\Sigma}=1\right)
$$

defines a family of unbiased estimators for $s^{2}$. We shall not consider the case where the scalars $x_{j}$ depend on the structural parameters. 


\subsection{Minimum-variance estimator under normal assumptions}

\subsubsection{Normal assumption}

We look for the minimum-variance estimator for $s^{2}$ in the family defined by (11). Unless new structural parameters are introduced, this problem is seen to have no solution under the general assumptions made hitherto. But it has a very simple one under the assumption that we introduce here.

\section{Normal assumption}

The conditional vector $X_{j}$, for $\Theta_{j}$ fixed, is normally distributed. Then it results from the general assumptions that this vector is $N\left(y \beta\left(\Theta_{j}\right), \sigma^{2}\left(\Theta_{j}\right) v_{j}\right)$. The following simple lemma is needed in section 6 .

\section{Lemma.}

Under the normal assumption, the conditional vector $\hat{b}_{j}$, for $\Theta_{j}$ fixed, is $N\left(\beta\left(\Theta_{j}\right), \sigma^{2}\left(\Theta_{j}\right) w_{j}\right)$.

\section{Demonstration}

By (4), $\hat{b}_{j}=u_{j} X_{j}$, with the obvious definition of $u_{j}\left(\begin{array}{l}t \\ g\end{array}\right)$.

Then

$$
\begin{aligned}
\operatorname{Cov}\left(\hat{b}_{j} / \Theta_{j}\right) & =u_{j}\left(\operatorname{Cov}\left(X_{j} / \Theta_{j}\right)\right) u_{j}^{\prime}=\sigma^{2}\left(\Theta_{j}\right) u_{j} v_{j} u_{j}^{\prime} \\
& =\sigma^{2}\left(\Theta_{j}\right) w_{j} .
\end{aligned}
$$

Note also that the relation $E\left(\hat{b}_{j} / \Theta_{j}\right)=\beta\left(\Theta_{j}\right)$ results from the regression assumption and (4).

Now the lemma results from the fact that a linearly transformed normal vector remains normal.

5.3.2. Calculation of $\operatorname{Var} \hat{s}^{2}$.

Theorem

Under the normal assumption,

$$
\operatorname{Var} \hat{s}^{2}=\left(\Sigma x_{j}^{2}\right)\left(s^{(4)}\left(1+\frac{2}{t-g}\right)-s^{4}\right),
$$

where $\hat{s}^{2}$ is defined by (11) and where

$$
s^{4}=\left(s^{2}\right)^{2}, s^{(4)}=E \sigma^{4}\left(\Theta_{j}\right)
$$

\section{Demonstration}

By the independence of the classes and the unbiasedness of $\hat{s}_{j}^{2}$,

$$
\begin{aligned}
\operatorname{Var} \hat{s}^{2}=\Sigma x_{j}^{2} \operatorname{Var} \hat{s}_{j}^{2} & =\Sigma x_{j}^{2}\left(E \dot{s}_{j}^{4}-E^{2} \hat{s}_{j}^{2}\right) \\
& =\Sigma x_{j}^{2}\left(E \hat{s}_{j}^{4}-s^{4}\right)
\end{aligned}
$$


By (4) and (10),

where

$$
(t-g) \hat{s}_{j}^{2}=X_{j}^{\prime} r_{j} X_{j}
$$

Then

$$
r_{j}=v_{j}^{-1}-v_{j}^{-1} y\left(y^{\prime} v_{j}^{-1} y\right)^{-1} y^{\prime} v_{j}^{-1},\left(\begin{array}{l}
t \\
t
\end{array}\right) .
$$

$$
\begin{gathered}
r_{j} y=0, r_{j} v_{j}=1^{\circ}-v_{j}^{-1} y\left(y^{\prime} v_{j}^{-1} y\right)^{-1} y^{\prime},\left(r_{j} v_{j}\right)^{2}=r_{j} v_{j}, \\
\operatorname{tr}\left(r_{j} v_{j}\right)=t-\operatorname{tr}\left(y^{\prime} v_{j}^{-1} y\left(y^{\prime} v_{j}^{-1} y\right)^{-1}\right)=t-\operatorname{tr} 1^{\circ 0}=t-g,
\end{gathered}
$$

where $1^{\circ}$ and $1^{\circ \circ}$ are respectively the $\left(\begin{array}{l}t \\ t\end{array}\right)$ and $\left(\begin{array}{l}g \\ g\end{array}\right)$ unit matrices and where we used the cyclical property of the trace.

Then, for $\Theta_{j}$ fixed, we have by $\left(\mathrm{A}_{4}\right)$ of the Appendix:

$$
\begin{aligned}
& (\mathrm{t}-\mathrm{g})^{2} E\left(\hat{s}_{j}^{4} / \Theta_{j}\right)=E\left(X_{j}^{\prime} r_{j} X_{j} X_{j}^{\prime} r_{j} X_{j} / \Theta_{j}\right)= \\
& \sigma^{4}\left(\Theta_{j}\right)\left(\operatorname{tr}^{2}\left(r_{j} v_{j}\right)+2 \operatorname{tr}\left(r_{j} v_{j}\right)^{2}\right)=\sigma^{4}\left(\Theta_{j}\right)\left((t-g)^{2}+2(t-g)\right) .
\end{aligned}
$$

Applying $E$ and using (14), relation (12) follows.

5.3.3. Minimum-variance-estimator

Theorem

Under the normal assumption, the minimum-variance estimator in the family (11) is

$$
\hat{\hat{s}}^{2}=\frac{1}{k} \Sigma \hat{s}_{j}^{2} .
$$

\section{Demonstration}

Results from (12) because the minimum of $\Sigma x_{j}^{2}$ under the constraint $x_{\Sigma}=1$ is obtained for $x_{1}=x_{2}=\ldots=x_{k}=1 / k$.

\section{Estimation OF THE COVARIANCE MATRIX}

\subsection{Fixed-class estimator}

\section{Theorem}

$$
\hat{a}_{j}=\left(\hat{b}_{j}-b\right)\left(\hat{b}_{j}-b\right)^{\prime}-s^{2} w_{j},\left(\begin{array}{l}
g \\
g
\end{array}\right)
$$

is an unbiased pseudo-estimator for the covariance matrix $a$.

\section{Demonstration}

The demonstration of the lemma in 5.3.1. shows that

$$
\operatorname{Cov}\left(\hat{b}_{j} / \Theta_{j}\right)=\sigma^{2}\left(\Theta_{j}\right) w_{j}, E\left(\hat{b}_{j} / \Theta_{j}\right)=\beta\left(\Theta_{j}\right) .
$$

(The normal assumption is not used at that stage). Then, because $E \hat{b}_{j}=b$ :

$$
E\left(\left(\hat{b}_{j}-b\right)\left(\hat{b}_{j}-b\right)^{\prime}\right)=\operatorname{Cov} \hat{b}_{j}=\operatorname{Cov} E\left(\hat{b}_{j} / \Theta_{j}\right)+E \operatorname{Cov}\left(\hat{b}_{j} / \Theta_{j}\right)
$$




$$
=\operatorname{Cov} \beta\left(\Theta_{j}\right)+E \sigma^{2}\left(\Theta_{j}\right) w_{j}=a+s^{2} w_{j} .
$$

The theorem is clear from this.

\subsection{Family of unbiased pseudo-estimators}

From the theorem results that

$$
\hat{a}=\Sigma x_{j} \hat{a}_{j}, \quad\left(x_{j}\left(\begin{array}{l}
g \\
g
\end{array}\right), x_{\Sigma}=1\right)
$$

defines a family of unbiased pseudo-estimators for the covariance matrix $a$. The matrix $a$ is symmetrical, whereas $\hat{a}$ furnished by (19) is not. Therefore NORBERG (1979) replaces $\hat{a}$ by

$$
\frac{1}{2}\left(\hat{a}+\hat{a}^{\prime}\right)
$$

(and also $b, s^{2}$ by estimators for these structural parameters).

We shall not perform the symmetrization at this stage. Of course, in practical work, any estimate for $a$ must finally be symmetrized.

6.3. Minimum-variance pseudo-estimator under normal and other assumptions 6.3.1. Notation.

We define

$$
a^{(2)}=E\left(\beta^{\circ}\left(\Theta_{j}\right) \beta^{\circ}\left(\Theta_{j}\right) \beta^{\circ}\left(\Theta_{j}\right) \beta^{\circ}\left(\Theta_{j}\right) .\right.
$$

Note that

$$
a=E\left(\beta^{\circ}\left(\Theta_{j}\right) \beta^{0^{\prime}}\left(\Theta_{j}\right)\right) .
$$

The notation $s^{(4)}$ already introduced in (13) is also used further.

$a^{(2)}$ and $s^{(4)}$ are fourth-order structural parameters.

\subsubsection{Independence assumption}

We shall use the assumption of independence of $\sigma^{2}\left(\Theta_{j}\right)$ and $\beta\left(\Theta_{j}\right)$. Briefly, we call it the independence assumption.

\subsubsection{Calculation of ScaVar $\hat{a}$.}

\section{Lemma}

Under the normal assumption (5.3.1.):

$$
\begin{aligned}
& E\left(\hat{b}_{j}^{\circ} x_{j}^{\prime} x_{j} \hat{b}_{j}^{\circ} \hat{b}_{j}^{\circ} \hat{b}_{j}^{\circ} / \Theta_{j}\right)= \\
& \sigma^{2}\left(\Theta_{j}\right) \operatorname{tr}\left(x_{j}^{\prime} x_{j} w_{j}\right) \operatorname{tr}\left(w_{j}\right)+2 \sigma^{4}\left(\Theta_{j}\right) \operatorname{tr}\left(x_{j}^{\prime} x_{j} w_{j} w_{j}\right) \\
& +4 \sigma^{2}\left(\Theta_{j}\right) \operatorname{tr}\left(x_{j}^{\prime} x_{j} w_{j} \beta^{\circ}\left(\Theta_{j}\right) \beta^{0^{\prime}}\left(\Theta_{j}\right)\right) \\
& +\sigma^{2}\left(\Theta_{j}\right) \operatorname{tr}\left(x_{j}^{\prime} x_{j} \beta^{\circ}\left(\Theta_{j}\right) \beta^{\circ}\left(\Theta_{j}\right)\right) \operatorname{tr}\left(w_{j}\right) \\
& +\sigma^{2}\left(\Theta_{j}\right) \operatorname{tr}\left(x_{j}^{\prime} x_{j} w_{j}\right) \operatorname{tr}\left(\beta^{\circ}\left(\Theta_{j}\right) \beta^{o^{\prime}}\left(\Theta_{j}\right)\right) \\
& +\operatorname{tr}\left(x_{j}^{\prime} x_{j} \beta^{\circ}\left(\Theta_{j}\right) \beta^{\circ{ }^{\prime}}\left(\Theta_{j}\right) \beta^{\circ}\left(\Theta_{j}\right) \beta^{\circ}\left(\Theta_{j}\right)\right) \text {. }
\end{aligned}
$$




\section{Demonstration}

By the lemma in 5.3.1, the conditional vector $\hat{b}_{j}^{\circ}$, for fixed $\Theta_{j}$ is $N\left(\beta^{\circ}\left(\Theta_{j}\right)\right.$, $\sigma^{2}\left(\Theta_{j}\right) w_{j}$ ). Then (21) follows from (A4) in the Appendix with

$$
a=x_{j}^{\prime} x_{j}, b=1, \mathrm{~m}=\beta^{\circ}\left(\Theta_{j}\right), v=\sigma^{2}\left(\Theta_{j}\right) w_{j} .
$$

Theorem

Under the normal (5.3.1) and independence (6.3.2) assumptions, for $\hat{a}$ defined by $(19)$ :

(22) ScaVar $\hat{\mathrm{a}}=\Sigma s^{(4)} \operatorname{tr}\left(x_{j}^{\prime} x_{j} w_{j}\right) \operatorname{tr}\left(w_{j}\right)+2 \Sigma s^{(4)} \operatorname{tr}\left(x_{j}^{\prime} x_{j} w_{j} w_{j}\right)$

$$
\begin{aligned}
& +{ }_{4} \Sigma s^{2} \operatorname{tr}\left(x_{j}^{\prime} x_{j} w_{j} a\right)+\Sigma s^{2} \operatorname{tr}\left(x_{j}^{\prime} x_{j} a\right) \operatorname{tr}\left(w_{j}\right) \\
& +\sum s^{2} \operatorname{tr}\left(x_{j}^{\prime} x_{j} w_{j}\right) \operatorname{tr} a+\Sigma \operatorname{tr}\left(x_{j}^{\prime} x_{j}(a)^{2}\right) \\
& -\Sigma \operatorname{tr}\left(x_{j}^{\prime} x_{j}\left(a+s^{2} w_{j}\right)\left(a+s^{2} w_{j}\right)\right) .
\end{aligned}
$$

\section{Demonstration}

Let $T\left(\begin{array}{l}g \\ g\end{array}\right)$ denote the pseudo-statistic

$$
T=\Sigma x_{j} \hat{b}_{j} \hat{b}_{j}^{\prime}=\Sigma x_{j}\left(\hat{b}_{j}-b\right)\left(\hat{b}_{j}-b\right)^{\prime},\left(\begin{array}{l}
g \\
g
\end{array}\right)
$$

Then

$$
\hat{a}=T-s^{2} \Sigma x_{j} w_{j} .
$$

Because the last term is non-random,

$$
\text { ScaVar } \hat{a}=\operatorname{ScaVar} T=\operatorname{tr} E\left(T T^{\prime}\right)-\operatorname{tr}\left(E T . E T^{\prime}\right) .
$$

Using the independence of classes,

$$
\begin{aligned}
E\left(T T^{\prime}\right) & =\sum_{i j} E\left(x_{i} \hat{b}_{i}^{0} \hat{b}_{i}^{\prime} \hat{b}_{j}^{0} \hat{b}_{j}^{o^{\prime}} x_{j}^{\prime}\right) \\
& =\sum_{,} E\left(x_{j} \hat{b}_{j}^{0} \hat{b}_{j}^{\circ} \hat{b}_{j}^{0} \hat{b}_{j}^{o^{\prime}} x_{j}^{\prime}\right)+\sum_{i \neq j} E\left(x_{i} \hat{b}_{i}^{0} \hat{b}_{i}^{b^{\prime}}\right) E\left(\hat{b}_{j}^{0} \hat{b}_{j}^{\circ} x_{j}^{\prime}\right) .
\end{aligned}
$$

Similarly,

(26) $E T \cdot E T^{\prime}=\sum_{j} E\left(x_{j} \hat{b}_{j} \hat{b}_{j}^{0_{j}^{\prime}}\right) E\left(\hat{b}_{j}^{0} \hat{b}_{j}^{o_{j}^{\prime}} x_{j}^{\prime}\right)+\sum_{i \neq j} E\left(x_{i} \hat{b}_{i}^{0} \hat{b}_{i}^{o^{\prime}}\right) E\left(\hat{b}_{j}^{0} \hat{b}_{j}^{o^{\prime}} x_{j}^{\prime}\right)$.

Thus, in the difference $E\left(T T^{\prime}\right)-E T . E T^{\prime}$, the sum in $i \neq j$ cancels.

By the cyclical property of the trace and the general relation $E()=$. $E E\left(. / \Theta_{j}\right)$, the trace of the first sum in the last member of (25) equals

$$
\Sigma E E\left(\hat{b}_{j}^{o} x_{j}^{\prime} x_{j} \hat{b}_{j}^{o} \hat{b}_{j}^{\circ} \hat{b}_{j}^{\circ} / \Theta_{j}\right)
$$

The positive terms in (22) result from this expression and from the lemma. The negative sum in (22) comes from the first sum in (26) after an application of (18). 
6.3.4. Minimum-variance pseudo-estimator.

Lemma

For $j=1,2, \ldots, k$ and $n=1,2, \ldots, r$, let $x_{j}$ be variable $\left(\begin{array}{l}g \\ g\end{array}\right)$ matrices, let $c_{n j}$ be fixed $\left(\begin{array}{l}g \\ g\end{array}\right)$ matrices and let $s_{n j}$ be fixed scalars. Then the extremum of

$$
\sum_{n j} s_{n j} \operatorname{tr}\left(x_{j}^{\prime} x_{j} c_{n j}\right),
$$

under the constraint $x_{\Sigma}=1$, is obtained for

$$
x_{j}=\bar{y}_{j} \text {, }
$$

where

$$
y_{j}=\underset{n}{\left(\sum s_{n j}\left(c_{n j}+c_{n j}^{\prime}\right)\right)^{-1}}
$$

\section{Demonstration}

Applying Lagrange's method, we consider $g^{2}$ multipliers $-\lambda_{p}^{q}$ corresponding to the constraints $x_{\Sigma}=1$. Expanding (27), we have to put equal to zero the partial derivatives in the variables of

$$
L=\sum_{n j \alpha \beta \gamma} s_{n j} x_{j \beta}^{\alpha} x_{j \beta}^{\gamma} c_{n j \gamma}^{\alpha}-\sum_{j p q} \lambda_{p}^{q} x_{j p}^{q} .
$$

Derivation in $x_{j p}^{q}$ gives the equation

$$
\sum_{n \gamma} s_{n j} x_{j p}^{\gamma} c_{n j \gamma}^{q}+\sum_{n \alpha} s_{n j} x_{j p}^{\alpha} c_{n j q}^{\alpha}=\lambda_{p}^{q}
$$

or, in matrix form

(30) $\sum_{n} s_{n j} x_{j} c_{n j}+\sum_{n} s_{n j} x_{j} c_{n j}^{\prime}=\lambda, x_{j}\left(\sum_{n} s_{n j}\left(c_{n j}+c_{n j}^{\prime}\right)\right)=\lambda, x_{j}=\lambda y_{j}$,

where $y_{j}$ is defined by (29). Summing over $j$, we obtain $1=\lambda y_{j}$. From this relation results $\lambda$. Substitution in (30) gives (28).

\section{Theorem}

Under the normal (5.3.1) and independence (6.3.2) assumptions, the minimumvariance pseudo-estimator in the family (19) is

$$
\hat{a}=\Sigma \bar{x}_{j} \hat{a}_{j}
$$

where

$$
x_{j}=a^{2}\left[\frac{3}{2}\left(s^{(4)}-s^{4}\right)\left(w_{j}\right)^{2}-\frac{1}{2}\left(3 a^{2}-a^{(2)}\right)+\left(a+s^{2} w_{j}\right)^{2}\right]^{-1} .
$$

For $g>1$, that result is based on the approximations (33).

\section{Demonstration}

By the lemma, the minimum of (22) is obtained for the matrices $\bar{x}_{j}$, where

$$
\begin{aligned}
& x_{j}^{-1}=2 s^{(4)} w_{j} \operatorname{tr} w_{j}+4 s^{(4)} w_{j} w_{j}+4 s^{2} w_{j} a+4 s^{2} a w_{j} \\
& \quad+2 s^{2} a \operatorname{tr} w_{j}+2 s^{2} w_{j} \operatorname{tr} a+2 a^{(2)}-2\left(a+s^{2} w_{j}\right)\left(a+s^{2} w_{j}\right) .
\end{aligned}
$$


Using the approximations (if $g>1$ )

$$
\left\{\begin{aligned}
w_{j} \operatorname{tr} w_{j}+2 w_{j} w_{j} \cong 3 w_{j} w_{j} \\
a \operatorname{tr} w_{j}+2 a w_{j} \cong 3 a w_{j} \\
w_{j} \operatorname{tr} a+2 w_{j} a 3 w_{j} a
\end{aligned}\right.
$$

one obtains

$$
x_{j}^{-1} \cong 6\left(s^{(4)}-s^{4}\right)\left(w_{j}\right)^{2}-2\left(3 a^{2}-a^{(2)}\right)+4\left(a+s^{2} w_{j}\right)^{2} .
$$

From this results (32), except for a constant (not depending on $j$ ) pre-factor. But such a pre-factor cancels in the formation of $\bar{x}_{j}$.

\section{Comments}

1. In any practical case, the quality of the approximations (33) can be tested on basis of the numerical data, at least with $a$ replaced by its estimate.

2. Let $q_{j}\left(\begin{array}{l}g \\ g\end{array}\right)$ be defined by

$$
\frac{3}{2}\left(s^{(4)}-s^{4}\right)\left(w_{j}\right)^{2}-\frac{1}{2}\left(3 a^{2}-a^{(2)}\right)+\left(a+s^{2} w_{j}\right)^{2}=\left(a+s^{2} w_{j}\right)^{2} q_{j} .
$$

Then, if $q_{j}=q$, independent of $j$, we are rid of the fourth-order structural parameters $s^{(4)}$ and $a^{(2)}$. Indeed, the inverse of the last member of (34) equals, in that case, $q^{-1}\left(a+s^{2} w_{j}\right)^{-2}$ and the constant pre-factor $q^{-1}$ cancels in the prenorming of these matrices. Then we obtain the following unbiased pseudoestimator for $a$ :

$$
\hat{\hat{a}}=\Sigma \bar{z}_{j}^{(2)} \hat{a}_{j}
$$

where

$$
z_{j}^{(2)}=a^{2}\left(a+s^{2} w_{j}\right)^{-2} .
$$

The irrelevant constant pre-factor $a^{2}$ is introduced again, because so $z_{j}^{(2)}=$ $\left(z_{j}\right)^{2}$ for $g=1$.

3. Les us now examine some cases where $q_{j}=q$, constant.

(i) Of course $q_{j}=q$ in the trivial case $w_{j}=w$, constant.

(ii) Let $\beta\left(\Theta_{j}\right)=b$ be degenerated. Then $a=(a)^{2}=0$ and in (34) each remaining term can be simplified by the factor $\left(w_{j}\right)^{2}$. Then $q_{j}=q$, constant.

(iii) Let $a^{(2)}=3 a^{2}$. For $g=1$, this means that the random variable $\beta\left(\Theta_{j}\right)$ has a coefficient of excess equal to zero. That is the case if it is normally distributed. Furthermore, assume the degeneracy $\sigma^{2}\left(\Theta_{j}\right)=s^{2}$. Then $s^{(4)}=s^{4}$ and $q_{j}=1$.

$\hat{a}(35)$

4. We summarize the arguments collected in favour of the pseudo-estimator

- It is unbiased, because $\bar{z}_{\Sigma}^{(2)}=1$.

- It does not depend on fourth-order structural parameters.

- It is optimal in degenerated situations. It will remain approximately optimal in cases close to such situations. It must be noted that it is 
precisely in case of the degeneracy described in (ii) that the estimation of $a$ is most delicate.

- For $g=1$, the weights $z_{j}^{(2)}$ are simply the squared credibility weights.

\subsection{Practical estimation of the covariance matrix}

\subsubsection{Problem.}

The pseudo-estimator $\hat{a}$ (35) and more generally, the pseudo-estimators (19), have a great inconvenience. Although they are unbiased when $b$ receives its true value in (16), they no longer have that interesting quality when $b$ is replaced, say by its optimal pseudo-estimator $\hat{\bar{b}}$ (9). But that is precisely what should be done in practice! The bias appears because $b$ does not figure linearly in the last member of (16). For $s^{2}$, there is no problem. Its replacement by $\hat{s}^{2}(15)$ causes no bias. For the structural parameters $a, s^{2}$ appearing in $z_{j}^{(2)}(36)$ the situation is different. Even when they are replaced by wrong estimates, $\overline{\hat{a}}(35)$ is unbiased because $\overline{z_{\Sigma}^{(2)}}=1$.

Thus, we have only to correct the situation for $b$. We shall start now from the pseudo-statistic

$$
\stackrel{T}{T}=\sum_{i} x_{j}\left(\hat{b}_{j}-\hat{b}\right)\left(\hat{b}_{j}-\hat{b}\right)^{\prime}\left(\begin{array}{l}
g \\
g
\end{array}\right),\left(x_{j}\left(\begin{array}{l}
g \\
g
\end{array}\right), x_{\Sigma}=1\right) .
$$

A look at (23) shows that $\hat{T}$ is $T$ wherein $b$ has been replaced by its optimal pseudo-estimator $\hat{b}$.

In case of $\hat{T}$, the research for optimal weights $x_{j}$ is based on so lengthy combinatorial calculations that it loses its interest.

In that respect, we shall simply transpose to the case of $\hat{T}$, the results obtained in section 6.3 .4 in the case of $T$. At least for $g=1$, lengthy arguments show that this is a good approximation.

6.4.2. A less "pseudo" family of unbiased pseudo-estimators for $a$.

\section{Theorem}

The relation

$$
\tilde{a}=\hat{T}-s^{2} \sum_{j} x_{j} w_{j}+z_{\Sigma}^{-1} a,\left(x_{j}\left(\begin{array}{l}
g \\
g
\end{array}\right), x_{\Sigma}=1\right),
$$

defines a family of unbiased pseudo-estimators for $a$.

\section{Demonstration}

We have

$$
\hat{T}=\sum_{i} x_{i}\left(\hat{b}_{i}^{\circ}-\sum_{j} \bar{z}_{j} \hat{b}_{j}\right)\left(\hat{b}_{i}^{\circ}-\sum_{k} \bar{z}_{k} \hat{b}_{k}^{\circ}\right)^{\prime}
$$


because it follows from $\bar{z}_{\Sigma}=1$ that the replacement of $\hat{b}_{i}$ by $\hat{b}_{i}^{o}$ is allowed. Then

$$
\hat{T}=\sum_{i} x_{i} \hat{b}_{i}^{\circ} \hat{b}_{i}^{\circ}+\sum_{\imath, k} x_{i} \bar{z}_{j} \hat{b}_{j} \hat{b}_{k}^{\circ} \bar{z}_{k}^{\prime \prime}-\sum_{\imath k} x_{i} z_{j} \hat{b}_{j} \hat{b}_{i}^{\prime}-\sum_{i k} x_{i} \hat{b}_{i} \hat{b}_{k}^{\circ \prime} z_{k}^{\prime} \text {. }
$$

Because $\Sigma x=1$, the $x_{i}$ can be summed out in the sum in $i j k$.

When we apply $E$, the dubble sums must be replaced by single sums and the indices must be egalized, say replaced by $j$. This follows from the fact that the $\hat{b}_{j}$ have zero expectation and are independent. By (18) we then obtain

$$
E \hat{T}=\sum_{i} x_{j}\left(a+s^{2} w_{j}\right)+\Sigma \bar{z}_{j}\left(a+s^{2} w_{j}\right) \bar{z}_{j}^{\prime}-\Sigma x_{j} \bar{z}_{j}\left(a+s^{2} w_{j}\right)-\Sigma x_{j}\left(a+s^{2} w_{j}\right) \bar{z}_{j}^{\prime}
$$

The first term in the last member equals $a+s^{2} \Sigma x_{i} w_{i}$.

From these consideration it is clear that

$$
\begin{aligned}
& \tilde{a}=\hat{T}-s^{2} \Sigma x_{j} w_{j}-\Sigma \bar{z}_{j}\left(a+s^{2} w_{j}\right) \bar{z}_{j} \\
& +\Sigma x_{j} \bar{z}_{j}\left(a+s^{2} w_{j}\right)+\Sigma x_{j}\left(a+s^{2} w_{j}\right) \bar{z}_{j}^{\prime},
\end{aligned}
$$

is an unbiased pseudo-estimator for $a$. From the relations

$$
\bar{z}_{j}=z_{\Sigma}^{-1} z_{j}=z_{\Sigma}^{-1} a\left(a+s^{2} w_{j}\right)^{-1}
$$

we obtain

$$
\Sigma \bar{z}_{j}\left(a+s^{2} w_{j}\right) \bar{z}_{j}^{\prime}=\Sigma z_{\Sigma}^{-1} a \bar{z}_{j}^{\prime}=z_{\Sigma}^{-1} a
$$

(showing in particular that $z_{\Sigma}^{-1} a$ is symmetrical) and similar relations allowing to simplify the last member of (39). Finally (38) is obtained.

\subsubsection{Practical estimation of $a$.}

Because a is symmetric, we adopt for it the pseudo-estimator (38) symmetrized in the obvious way. As matrical weights $x_{j}$ in (38) we suggest to take the prenormed $z_{j}^{(2)}$ defined by (36), i.e. the same weights $\bar{z}_{j}^{(2)}$ as in (35).

7. Summary of all steps to BE EXECUted for the calculation of the CREDIBILITY ESTIMATORS

Steps depending on $j$ must be executed for $j=1,2, \ldots, k$.

o) Given: $X_{j}\left(\begin{array}{l}1 \\ t\end{array}\right), v_{j}\left(\begin{array}{l}t \\ t\end{array}\right), y\left(\begin{array}{c}g \\ t\end{array}\right)$

1) Calculate $w_{j}=\left(y^{\prime} v_{j}^{-1} y\right)^{-1}\left(\begin{array}{c}g \\ g\end{array}\right)$

2) Calculate $\hat{b}_{j}=w_{j} y^{\prime} v_{j} X_{j}\left(\begin{array}{c}1 \\ g\end{array}\right)$

3) Calculate the scalar $s^{2}=\frac{1}{k(t-g)} \Sigma\left(X_{j}-y \hat{b}_{j}\right)^{\prime} v_{j}^{-1}\left(X_{j}-y \hat{b}_{j}\right)$

4) Fix initial $a=\operatorname{diag}(c, c, \ldots, c)\left(\begin{array}{l}g \\ g\end{array}\right)$, (c large) 
5) Calculate $z_{j}=a\left(a+s^{2} w_{j}\right)^{-1}\left(\begin{array}{l}g \\ g\end{array}\right), z_{\Sigma}\left(\begin{array}{l}g \\ g\end{array}\right), \bar{z}_{j}\left(\begin{array}{l}g \\ g\end{array}\right)$, $z_{j}^{(2)}=a^{2}\left(a+s^{2} w_{j}\right)^{-2}\left(\begin{array}{l}g \\ g\end{array}\right), z_{\Sigma}^{(2)}\left(\begin{array}{l}g \\ g\end{array}\right), x_{j}=\bar{z}_{j}^{(2)}\left(\begin{array}{l}g \\ g\end{array}\right)$

6) Calculate $b=\Sigma \bar{z}_{j} \hat{b}_{j}\left(\begin{array}{l}\mathbf{l} \\ g\end{array}\right)$

7) Calculate $\mathrm{S}=\Sigma x_{j}\left(\hat{b}_{j}-b\right)\left(\hat{b}_{j}-b\right)^{\prime}-s^{2} \Sigma x_{j} w_{j}+z_{\Sigma}^{-1} a\left(\begin{array}{c}\boldsymbol{g} \\ \boldsymbol{g}\end{array}\right)$

8) Calculate New $a=\frac{1}{2}\left(S+S^{\prime}\right)\left(\begin{array}{l}g \\ g\end{array}\right)$

9) Return to step 5) with $a$ replaced by New $a$, as many times as necessary, until $a$ is stabilized.

10) Calculate $B_{j}=\left(1-z_{j}\right) b+z_{j} \hat{b}_{j}$.

APPENDIX

Expectation of products of quadratic forms in normal variables

\section{Theorem}

Let $a, b, v$ be symmetrical $\left(\begin{array}{l}t \\ t\end{array}\right)$ matrices, $v$ positive definite. If the $\left(\begin{array}{l}1 \\ t\end{array}\right)$ vector $X$ is $N(0, v)$, then

$$
\begin{gathered}
E\left(X^{\prime} a X\right)=\operatorname{tr}(a v), \\
E\left(X^{\prime} a X X^{\prime} b X\right)=\operatorname{tr}(a v) \operatorname{tr}(b v)+2 \operatorname{tr}(a v b v)
\end{gathered}
$$

If $X$ is $N(m, v)$, then

$$
\begin{gathered}
E\left(X^{\prime} a X\right)=\operatorname{tr}(a v)+\operatorname{tr}\left(a m m^{\prime}\right) \\
E\left(X^{\prime} a X X^{\prime} b X\right)=\operatorname{tr}(a v) \operatorname{tr}(b v)+2 \operatorname{tr}(a v b v)+4 \operatorname{tr}\left(a v b m m^{\prime}\right) \\
+\operatorname{tr}\left(a m m^{\prime}\right) \operatorname{tr}(b v)+\operatorname{tr}\left(b m m^{\prime}\right) \operatorname{tr}(a v)+\operatorname{tr}\left(a m m^{\prime} b m m^{\prime}\right) .
\end{gathered}
$$

\section{Demonstration}

$$
E\left(X^{\prime} a X\right)=\sum_{i, j} E\left(X_{i} a_{i}^{j} X_{j}\right)=\sum_{i, j} a_{i}^{j} E\left(X_{i} X_{j}\right)=\sum_{i, 1} a_{i}^{j} v_{j}^{i}=\operatorname{tr}(a v)
$$

(A2) Let the $\left(\begin{array}{l}1 \\ t\end{array}\right)$ vector $Y$ be $N(0,1)$. Then each component is normal with zero mean and unit variance. Therefore

$$
E Y_{i}=o, E Y_{i}^{2}=1, E Y_{i}^{3}=0, E Y_{i}^{4}=3 \text {. }
$$

Moreover, $Y_{1}, Y_{2}, \ldots, Y_{t}$ are independent. From these facts follows the combinatorial formula

$$
E\left(Y_{i} Y_{j} Y_{k} Y_{l}\right)=\delta_{i j} \delta_{k l}+\delta_{i k} \delta_{l j}+\delta_{i l} \delta_{j k}
$$

where $\delta_{i j}$ is Kronecker's symbol.

Indeed, if $m, n, p, q$ are 4 different indices, the possible patterns for $i, j, k, l$ are

$$
\begin{array}{l|lllllllllll}
i & m & m & n & n & n & m & m & m & m & \ldots & m \\
j & m & n & m & n & n & m & n & n & m & \ldots & n \\
k & m & n & n & m & n & n & m & n & n & \ldots & p \\
l & m & n & n & n & m & n & n & m & p & \ldots & q
\end{array}
$$


and for each pattern, the verification of (A5) is immediate. Then

$$
\begin{gathered}
E\left(Y^{\prime} a Y Y^{\prime} b Y\right)=\sum_{i j k l} E\left(Y_{i} a_{i}^{j} Y_{j} Y_{k} b_{k}^{l} Y_{1}\right)=\sum_{i j k l} a_{i}^{j} b_{k}^{l} E\left(Y_{i} Y_{j} Y_{k} Y_{l}\right)= \\
\sum_{i j k l} a_{i}^{j} b_{k}^{l}\left(\delta_{i j} \delta_{k l}+\delta_{i k} \delta_{l j}+\delta_{i l} \delta_{j k}\right)=\sum_{i k} a_{i}^{i} b_{k}^{k}+\sum_{i l} a_{i}^{l} b_{i}^{l}+\sum_{i j} a_{i}^{j} b_{j}^{i}= \\
\sum_{i} a_{i}^{i} \Sigma b_{k}^{k}+\sum_{i l} a_{l}^{l} b_{l}^{i}+\sum_{i j} a_{i}^{j} b_{j}^{i}=\operatorname{tr} \cdot a \cdot \operatorname{tr} b+\operatorname{tr}(a b)+\operatorname{tr}(a b) .
\end{gathered}
$$

This proves (A2) for $v=1$.

Now we prove $(\mathrm{A} 2)$ for $X$ supposed to be $N(\mathrm{o}, v)$. It is classical that for some $\left(\begin{array}{l}t \\ t\end{array}\right)$ matrix $s$, we have $X=s Y$ with $Y N(\mathrm{o}, 1)$.

Then

$$
v=\operatorname{Cov} X=s(\operatorname{Cov} Y) s^{\prime}=s s^{\prime}
$$

and

$$
\begin{aligned}
E\left(X^{\prime} a X X^{\prime} b X\right) & =E\left(Y^{\prime} s^{\prime} a s Y Y^{\prime} s^{\prime} b s Y\right) \\
& =\operatorname{tr}\left(s^{\prime} a s\right) \operatorname{tr}\left(s^{\prime} b s\right)+2 \operatorname{tr}\left(s^{\prime} a s s^{\prime} b s\right) \\
& =\operatorname{tr}\left(a s s^{\prime}\right) \operatorname{tr}\left(b s s^{\prime}\right)+2 \operatorname{tr}\left(a s s^{\prime} b s s^{\prime}\right) \\
& =\operatorname{tr}(a v) \operatorname{tr}(b v)+2 \operatorname{tr}(a v b v),
\end{aligned}
$$

by the cyclical property of the trace.

(A3) We decompose $X=Y+m$, where $Y=X-m$ is $N(\mathrm{o}, v)$.

\section{Then}

$$
X^{\prime} a X=Y^{\prime} a Y+2 Y^{\prime} a m+m^{\prime} a m
$$

and $\left(\mathrm{A}_{3}\right)$ follows from $\left(\mathrm{A}_{1}\right)$ and the relations

$$
E\left(Y^{\prime} a m\right)=0, \quad m^{\prime} a m=\operatorname{tr}\left(m^{\prime} a m\right)=\operatorname{tr}\left(a m m^{\prime}\right) .
$$

(A4) For (A4) we use (A6) and the similar decomposition

$$
X^{\prime} b X=Y^{\prime} b Y+2 m^{\prime} b Y+m^{\prime} b m \text {. }
$$

The expectation of the product of (A6) and $\left(A_{7}\right)$ is best displayed in a table. Making use of (A1), (A2) we obtain the table

\begin{tabular}{c|ccc} 
& $Y^{\prime} b Y$ & $2 m^{\prime} b Y$ & $m^{\prime} b m$ \\
\hline$Y^{\prime} a Y$ & $\begin{array}{c}\operatorname{tr}(a v) \operatorname{tr}(b v) \\
+2 \operatorname{tr}(a v b v)\end{array}$ & 0 & $\operatorname{tr}(a v) m^{\prime} b m$ \\
$2 Y^{\prime} a m$ & 0 & $4 \operatorname{tr}(a m m, b v)$ & 0 \\
$m^{\prime} a m$ & $m^{\prime} a m t r(b v)$ & 0 & $m^{\prime} a m m^{\prime} b m$
\end{tabular}

After slight transformations (use cyclical property of trace, and also that a matrix and its transposed matrix have the same trace), formula (A4) results from this table. However, some supplementary explanations may be useful. 
First, the matrix $a m m^{\prime} b$ is not necessarily symmetrical. The corresponding term $4 \operatorname{tr}\left(a m m^{\prime} b v\right)$ is the right one because (AI) is valid even if $a$ is not symmetrical (see demonstration). We have also to explain the zero's in the table. For instance, let us justify the relation

$$
E\left(Y^{\prime} a Y m^{\prime} b Y\right)=o .
$$

For some $\left(\begin{array}{l}t \\ t\end{array}\right)$ matrix $s, Y$ can be expressed as $Y=s Z$ where the $\left(\begin{array}{l}1 \\ t\end{array}\right)$ vector $Z$ is $N(0,1)$. Then the components $Z_{i}$ of $Z$ are independent and have zero expectation. The development of $Y^{\prime} a Y m^{\prime} b Y$ is an expression $\Sigma c_{i j k} Z_{i} Z_{j} Z_{k}$. Among the indices $i, j, k$, at least one must appear an odd number of times. Therefore $E\left(Z_{i} Z_{j} Z_{k}\right)=o$.

\section{REFERENCES}

Bühlmann, H. and Straub, E. (1970). Glaubwürdigkeit für Schadensätze. Mitt. Ver. Schw. Vers.-Math., 79, 111-133.

De Vyider, F. (1976). Geometrical Credibility. Scandinavian Actuarial Journal, 1976, $121-149$.

De Vylder, F. (1978). Parameter estimation in credibility theory. Astin Bulletin, 10, 99-112.

HACHEMEISTER, C. (1975). Credibility for regression models with application to trend. In Kahn (1975).

Kahn, P. M., Editor. (1975). Credibility. Theory and Applications. (Academic Press). NorberG, R. (1979). The credibility approach to experience rating. Scandinavian Actuarial Journal, 1979, 181-221.

Norberg, R. (1980). Emperical Bayes credibility. Scandinavian Actuarial Journal, 1980, 177-194.

TAyLOR, G. C. (1977). Abstract Credibility. Scandinavian Actuarial Journal, 1977 149-168. 